\title{
Synthesis and biological activity evaluation Of 3-[2-(1h-imidazol-2-Yl)alkyl]- 2-thioxo-2,3-dihydroquinazolin-4 (1h) -one derivatives
}

\author{
Oksana O. Zavada', Olena V. Tkachenko², Volodymyr V. Kazmirchuk, \\ Iryna O. Zhuravel ${ }^{4}$, Vyacheslav O. Lebedynets ${ }^{2}$, O. Proskurova la $^{5}$
}

${ }^{1}$ Department of Medical and Bioorganic Chemistry, Kharkiv National Medical University, Ukraine, ${ }^{2}$ Department of Quality Management, National University of Pharmacy, Ukraine, ${ }^{3}$ Antibacterial Agents Laboratory,

Mechnikov Institute of Microbiology and Immunology Ukraine, ${ }^{4}$ Department of Clinical Biochemistry, Forensic

Toxicology and Pharmacy, Kharkiv Medical Academy of Postgraduate Education, ${ }^{5}$ Department of Social

Pharmacy, National University of Pharmacy

\section{Abstract}

Aims: Detection of general patterns of the synthesis of quinazoline derivatives contains a fragment of 2-aminoalkylimidazole and studying their antimicrobial activity. Materials and Methods: Methods of organic synthesis; physical and physicochemical methods of analysis of organic compounds ${ }^{1}$ Hydrogen nuclear magnetic resonance spectroscopy and elemental analysis were used. Results: To construct a focused library of compounds with potential antimicrobial and antifungal properties, we have chosen a strategy of combining quinazoline fragments with an imidazole residue in one molecule. The possibility of using 2-aminoalkylimidazoles as an amine component in the heterocyclization reaction with o-isothiocyanato esters was considered. 3-Substituted 2-thioxoquinazoline-4-ones were synthesized by the interaction of methyl esters of 4,5-substituted 2 -isothiocyanatobenzoic acids with $2-(\alpha, \beta, \omega$-aminoalkyl)imidazoles. Experimental study of antimicrobial activity was performed for the obtained substances, which according to the results of virtual screening showed the best results. Conclusions: A virtual library design with structural fragments of quinazoline and imidazole was made. The systematic series of 6,7-substituted 3-[2-(1H-imidazol-2-yl)-alkyl]-2-thioxo-2,3-dihydroquinazolin-4(1H)one were synthesized. According to the results of the study of the biological effects of the new derivatives of 3-N-(alkylimidazolyl-2) pyrimidine, a number of patterns of connection "chemical structure - antibacterial action" were established and the main directions of the purposeful modification of the structure for the search of new antimicrobial and antifungal agents were determined.

Key words: 2 - $(\alpha, \beta, \omega$-aminoalkyl)imidazole, 2 -isothiocyanatobenzoate, 3-[2-(1H-imidazol-2-yl)alkyl]-2-thioxo2,3-dihydroquinazolin-4(1H)-one, antibacterial activity, antifungal activity

\section{INTRODUCTION}

$\mathrm{R}$ ecently compounds, which combine several heterocyclic fragments in their structures, attract particular interest in the development of innovative drug substances. The combination of various pharmacophores, especially heterocyclic fragments, in the same molecule, can lead both to the synergy of known effects and the emergence of new kinds of pharmacological activity. The choice of pharmacophore fragments for modeling of new structures is based on many factors, among which the most important is the analysis of the evidencebased medicine data about pharmacological action of substances containing the fragment. In this aspect, derivatives of imidazole and quinazoline are promising compounds, because these fragments are part of the structure of known antimicrobial drugs ${ }^{[1-3]}$ and substances, which demonstrated antitubercular ${ }^{[4]}$ and antimicrobial ${ }^{[5-10]}$ activity

Address for correspondence:

Oksana O. Zavada, Department of Medical and

Bioorganic Chemistry, Kharkiv National Medical

University, Ukraine, E-mail: o.o.zavada@ukr.net

Received: $10-10-2018$

Revised: 23-11-2018

Accepted: 01-12-2018 
during preclinical trials. However, it should be noted that there is almost no information about the synthesis and properties of quinazolines containing 2-aminoalkylimidazole moiety. Hence, revealing general pattern about the synthesis of quinazolines, combined by alkyl chain with 2-aminoalkylimidazole moiety and examination of their pharmacological potential is actual and represent the subject of the present study.

\section{MATERIALS AND METHODS}

A number of virtual screening methods have been applied to estimate potential biologic activity. Computer modeling of a virtual library of quinazoline derivatives, which contains 2-aminoalkylimidazole moiety, has been carried out by Project Library software. For the design of virtual library of 3-[2-(1H-imidazol-2-yl)alkyl]-2-thioxo-2,3dihydroquinazolin-4(1H)-ones 4 randomization points (length of the alkyl chain, substituents in imidazole, alkyl chain, 6 or 7 position of quinazoline moiety) was chosen based on availability of starting 2-aminobenzoates and in attempts to achieve chemical diversity in imidazole moiety and alkyl chain. Evaluation of potential biologic activity has been conducted by PASS Professional, version 2010. ${ }^{[1]}$ This determined the path of synthesis and the most promising compounds were synthesized. Analysis of the computer prediction results for a virtual library of 3-[2-(1H-imidazol2-yl)alkyl]-2-thioxo-2,3-dihydroquinazolin-4( $1 H$ )ones showed high level of probability of antimicrobial and antifungal activity and allowed to generate the library of the most perspective compounds 4 $\{1-30\}$ for further biological investigations [Table 1].

\begin{tabular}{|c|c|c|c|c|c|}
\hline Compounds & $n$ & $\mathbf{R}$ & Molecular formula, m. m. & Yield, \% & $n, \%$ calc. exp. \\
\hline $4\{1\}$ & $n=0$ & $\mathrm{R}_{1}=\mathrm{H}, \mathrm{R}_{2}=\mathrm{H}, \mathrm{R}_{3}=\mathrm{H}, \mathrm{R}_{4}=\mathrm{H}$ & $\mathrm{C}_{12} \mathrm{H}_{10} \mathrm{~N}_{4} \mathrm{OS} 258.30$ & 82 & $21.69(21.72)$ \\
\hline $4\{2\}$ & $n=1$ & $\mathrm{R}_{1}=\mathrm{H}, \mathrm{R}_{2}=\mathrm{H}, \mathrm{R}_{3}=\mathrm{H}, \mathrm{R}_{4}=\mathrm{H}$ & $\mathrm{C}_{13} \mathrm{H}_{12} \mathrm{~N}_{4} \mathrm{OS} 272.33$ & 85 & $20.57(20.59)$ \\
\hline $4\{3\}$ & $n=2$ & $\mathrm{R}_{1}=\mathrm{H}, \mathrm{R}_{2}=\mathrm{H}, \mathrm{R}_{3}=\mathrm{H}, \mathrm{R}_{4}=\mathrm{H}$ & $\mathrm{C}_{14} \mathrm{H}_{14} \mathrm{~N}_{4} \mathrm{OS} 286.36$ & 88 & $19.57(19.60)$ \\
\hline $4\{4\}$ & $n=3$ & $\mathrm{R}_{1}=\mathrm{H}, \mathrm{R}_{2}=\mathrm{H}, \mathrm{R}_{3}=\mathrm{H}, \mathrm{R}_{4}=\mathrm{H}$ & $\mathrm{C}_{15} \mathrm{H}_{16} \mathrm{~N}_{4} \mathrm{OS} 300.38$ & 81 & $18.65(18.69)$ \\
\hline $4\{5\}$ & $n=0$ & $\mathrm{R}_{1}=\mathrm{H}, \mathrm{R}_{2}=\mathrm{H}, \mathrm{R}_{3}=\mathrm{CH}_{3}, \mathrm{R}_{4}=\mathrm{H}$ & $\mathrm{C}_{13} \mathrm{H}_{12} \mathrm{~N}_{4} \mathrm{OS} 272.33$ & 84 & $20.57(20.61)$ \\
\hline $4\{6\}$ & $n=0$ & $\mathrm{R}_{1}=\mathrm{H}, \mathrm{R}_{2}=\mathrm{H}, \mathrm{R}_{3}=\mathrm{CH}\left(\mathrm{CH}_{3}\right)_{2}, \mathrm{R}_{4}=\mathrm{H}$ & $\mathrm{C}_{15} \mathrm{H}_{16} \mathrm{~N}_{4} \mathrm{OS} 300.38$ & 83 & $18.65(18.700$ \\
\hline $4\{7\}$ & $n=0$ & $\mathrm{R}_{1}=\mathrm{H}, \mathrm{R}_{2}=\mathrm{H}, \mathrm{R}_{3}=\mathrm{CH}_{2} \mathrm{CH}\left(\mathrm{CH}_{3}\right)_{2}, \mathrm{R}_{4}=\mathrm{H}$ & $\mathrm{C}_{16} \mathrm{H}_{18} \mathrm{~N}_{4} \mathrm{OS} 314.41$ & 87 & $17.8(17.86)$ \\
\hline $4\{8\}$ & $n=0$ & $\mathrm{R}_{1}=\mathrm{H}, \mathrm{R}_{2}=\mathrm{H}, \mathrm{R}_{3}=\mathrm{CH}\left(\mathrm{CH}_{3}\right) \mathrm{CH}_{5}, \mathrm{R}_{4}=\mathrm{H}$ & $\mathrm{C}_{16} \mathrm{H}_{18} \mathrm{~N}_{4} \mathrm{OS} 314.41$ & 82 & $17.82(17.87)$ \\
\hline $4\{9\}$ & $n=0$ & $\mathrm{R}_{1}=\mathrm{H}, \mathrm{R}_{2}=\mathrm{H}, \mathrm{R}_{3}=\mathrm{Bn}, \mathrm{R}_{4}=\mathrm{H}$ & $\mathrm{C}_{19} \mathrm{H}_{16} \mathrm{~N}_{4} \mathrm{OS} 348.43$ & 84 & $16.08(16.11)$ \\
\hline $4\{10\}$ & $n=1$ & $\mathrm{R}_{1}=\mathrm{H}, \mathrm{R}_{2}=\mathrm{H}, \mathrm{R}_{3}=\mathrm{H}, \mathrm{R}_{4}=\mathrm{Bn}$ & $\mathrm{C}_{20} \mathrm{H}_{18} \mathrm{~N}_{4} \mathrm{OS} 362.46$ & 83 & $15.46(15.48)$ \\
\hline $4\{11\}$ & $n=0$ & $\mathrm{R}_{1}=\mathrm{CH} 3, \mathrm{R}_{2}=\mathrm{H}, \mathrm{R}_{3}=\mathrm{H}, \mathrm{R}_{4}=\mathrm{H}$ & $\mathrm{C}_{13} \mathrm{H}_{12} \mathrm{~N}_{4} \mathrm{OS} 272.33$ & 90 & $20.57(20.60)$ \\
\hline $4\{12\}$ & $n=1$ & $\mathrm{R}_{1}=\mathrm{CH}_{3}, \mathrm{R}_{2}=\mathrm{H}, \mathrm{R}_{3}=\mathrm{H}, \mathrm{R}_{4}=\mathrm{H}$ & $\mathrm{C}_{14} \mathrm{H}_{14} \mathrm{~N}_{4} \mathrm{OS} 286.36$ & 87 & $19.57(19.60)$ \\
\hline $4\{13\}$ & $n=2$ & $\mathrm{R}_{1}=\mathrm{CH}_{3}, \mathrm{R}_{2}=\mathrm{H}, \mathrm{R}_{3}=\mathrm{H}, \mathrm{R}_{4}=\mathrm{H}$ & $\mathrm{C}_{15} \mathrm{H}_{16} \mathrm{~N}_{4} \mathrm{OS} 300.38$ & 85 & $18.65(18.68)$ \\
\hline $4\{14\}$ & $n=3$ & $\mathrm{R}_{1}=\mathrm{CH}_{3}, \mathrm{R}_{2}=\mathrm{H}, \mathrm{R}_{3}=\mathrm{H}, \mathrm{R}_{4}=\mathrm{H}$ & $\mathrm{C}_{16} \mathrm{H}_{18} \mathrm{~N}_{4} \mathrm{OS} 314.41$ & 88 & $17.82(17.84)$ \\
\hline $4\{15\}$ & $n=0$ & $\mathrm{R}_{1}=\mathrm{CH}_{3}, \mathrm{R}_{2}=\mathrm{H}, \mathrm{R}_{3}=\mathrm{CH} 3, \mathrm{R}_{4}=\mathrm{H}$ & $\mathrm{C}_{16} \mathrm{H}_{18} \mathrm{~N}_{4} \mathrm{OS} 314.41$ & 86 & $17.82(17.84)$ \\
\hline $4\{16\}$ & $n=0$ & $\mathrm{R}_{1}=\mathrm{CH}_{3}, \mathrm{R}_{2}=\mathrm{H}, \mathrm{R}_{3}=\mathrm{CH}\left(\mathrm{CH}_{3}\right)_{2}, \mathrm{R}_{4}=\mathrm{H}$ & $\mathrm{C}_{14} \mathrm{H}_{14} \mathrm{~N}_{4} \mathrm{OS} 286.36$ & 81 & $19.57(19.60)$ \\
\hline $4\{17\}$ & $n=0$ & $\mathrm{R}_{1}=\mathrm{CH}_{3}, \mathrm{R}_{2}=\mathrm{H}, \mathrm{R}_{3}=\mathrm{CH}_{2} \mathrm{CH}\left(\mathrm{CH}_{3}\right)_{2}, \mathrm{R}_{4}=\mathrm{H}$ & $\mathrm{C}_{17} \mathrm{H}_{20} \mathrm{~N}_{4} \mathrm{OS} 328.44$ & 80 & $17.0617 .10)$ \\
\hline $4\{18\}$ & $n=0$ & $\mathrm{R}_{1}=\mathrm{CH}_{3}, \mathrm{R}_{2}=\mathrm{H}, \mathrm{R}_{3}=\mathrm{CH}\left(\mathrm{CH}_{3}\right) \mathrm{C}_{2} \mathrm{H}_{5}, \mathrm{R}_{4}=\mathrm{H}$ & $\mathrm{C}_{17} \mathrm{H}_{20} \mathrm{~N}_{4} \mathrm{OS} 328.44$ & 82 & $17.0(17.10)$ \\
\hline $4\{19\}$ & $n=0$ & $\mathrm{R}_{1}=\mathrm{CH}_{3}, \mathrm{R}_{2}=\mathrm{H}, \mathrm{R}_{3}=\mathrm{Bn}, \mathrm{R}_{4}=\mathrm{H}$ & $\mathrm{C}_{20} \mathrm{H}_{18} \mathrm{~N}_{4} \mathrm{OS} 362.46$ & 91 & $15.46(15.46)$ \\
\hline $4\{20\}$ & $n=1$ & $\mathrm{R}_{1}=\mathrm{CH}_{3}, \mathrm{R}_{2}=\mathrm{H}, \mathrm{R}_{3}=\mathrm{H}, \mathrm{R}_{4}=\mathrm{Bn}$ & $\mathrm{C}_{21} \mathrm{H}_{20} \mathrm{~N}_{4} \mathrm{OS} 376.48$ & 92 & $14.88(14.88)$ \\
\hline $4\{21\}$ & $n=1$ & $\mathrm{R}_{1}=\mathrm{F}, \mathrm{R}_{2}=\mathrm{H}, \mathrm{R}_{3}=\mathrm{H}, \mathrm{R}_{4}=\mathrm{H}$ & $\mathrm{C}_{13} \mathrm{H}_{11} \mathrm{FN}_{4} \mathrm{O} 290.32$ & 90 & $19.30(19.35)$ \\
\hline $4\{22\}$ & $n=2$ & $\mathrm{R}_{1}=\mathrm{F}, \mathrm{R}_{2}=\mathrm{H}, \mathrm{R}_{3}=\mathrm{H}, \mathrm{R}_{4}=\mathrm{H}$ & $\mathrm{C}_{14} \mathrm{H}_{13} \mathrm{FN}_{4} \mathrm{OS} 304.35$ & 86 & $18.41(18.45)$ \\
\hline $4\{23\}$ & $n=3$ & $\mathrm{R}_{1}=\mathrm{F}, \mathrm{R}_{2}=\mathrm{H}, \mathrm{R}_{3}=\mathrm{H}, \mathrm{R}_{4}=\mathrm{H}$ & $\mathrm{C}_{15} \mathrm{H}_{15} \mathrm{FN}_{4} \mathrm{OS} 318.38$ & 92 & $17.60(17.64)$ \\
\hline $4\{24\}$ & $n=1$ & $\mathrm{R}_{1}=\mathrm{F}, \mathrm{R}_{2}=\mathrm{H}, \mathrm{R}_{3}=\mathrm{H}, \mathrm{R}_{4}=\mathrm{Bn}$ & $\mathrm{C}_{20} \mathrm{H}_{17} \mathrm{FN}_{4} \mathrm{OS} 380.45$ & 93 & $14.73(14.79)$ \\
\hline $4\{25\}$ & $n=1$ & $\mathrm{R}_{1}=\mathrm{Cl}, \mathrm{R}_{2}=\mathrm{H}, \mathrm{R}_{3}=\mathrm{H}, \mathrm{R}_{4}=\mathrm{H}$ & $\mathrm{C}_{13} \mathrm{H}_{11} \mathrm{CIN}_{4} \mathrm{OS} 306.78$ & 87 & $18.26(18.30)$ \\
\hline $4\{26\}$ & $n=2$ & $\mathrm{R}_{1}=\mathrm{Cl}, \mathrm{R}_{2}=\mathrm{H}, \mathrm{R}_{3}=\mathrm{H}, \mathrm{R}_{4}=\mathrm{H}$ & $\mathrm{C}_{14} \mathrm{H}_{13}$ CIN4OS 320.80 & 88 & $17.46(17.48)$ \\
\hline $4\{27\}$ & $n=3$ & $\mathrm{R}_{1}=\mathrm{Cl}, \mathrm{R}_{2}=\mathrm{H}, \mathrm{R}_{3}=\mathrm{H}, \mathrm{R}_{4}=\mathrm{H}$ & $\mathrm{C}_{15} \mathrm{H}_{15} \mathrm{CIN}_{4} \mathrm{OS} 334.83$ & 86 & $16.73(16.75)$ \\
\hline $4\{28\}$ & $n=1$ & $\mathrm{R}_{1}=\mathrm{H}, \mathrm{R}_{2}=\mathrm{Cl}, \mathrm{R}_{3}=\mathrm{H}, \mathrm{R}_{4}=\mathrm{Bn}$ & $\mathrm{C}_{20} \mathrm{H}_{17} \mathrm{CIN}_{4} \mathrm{OS} 396.90$ & 96 & $14.12(14.15)$ \\
\hline $4\{29\}$ & $n=1$ & $\mathrm{R}_{1}=\mathrm{H}, \mathrm{R}_{2}=\mathrm{COOCH}_{3}, \mathrm{R}_{3}=\mathrm{H}, \mathrm{R}_{4}=\mathrm{H}$ & $\mathrm{C}_{15} \mathrm{H}_{14} \mathrm{~N}_{4} \mathrm{O}_{3} \mathrm{~S} 330.37$ & 85 & $16.96(16.98)$ \\
\hline $4\{30\}$ & $n=2$ & $\mathrm{R}_{1}=\mathrm{H}, \mathrm{R}_{2}=\mathrm{COOCH}_{3}, \mathrm{R}_{3}=\mathrm{H}, \mathrm{R}_{4}=\mathrm{H}$ & $\mathrm{C}_{16} \mathrm{H}_{16} \mathrm{~N}_{4} \mathrm{O}_{3} \mathrm{~S} 344.39$ & 84 & $16.27(16.30)$ \\
\hline
\end{tabular}


In continuation of investigations conducting at the National University of Pharmacy, which lead to suitable synthetic methods, providing high yields and purity of 3 -substituted condensed 2-thioxopyrimidin-4-ones, ${ }^{[12-15]}$ we examined the possibility of use $2-(\alpha, \beta, \omega$-aminoalkyl) imidazoles as amine components due to presence of primary amino group in the heterocyclization reaction with ortho-isothiocyanato esters. Synthesis of intermediate methyl 2-isothiocyanatobenzoates 2\{1-6\} was carried out by treatment of substituted methyl 2-aminobenzoates $1\{1$ 6 \} with thiophosgene in a two-phase system of chloroform - water at room temperature [Figure 1].

Starting 2-( $\alpha, \beta, \omega$-aminoalkyl)imidazoles $3\{1-10\}$ wasobtained according to previously developed methods. ${ }^{[16-17]} 3$-Subsituted 2-thioxoquinazolin-4-ones $4\{1-30\}$ was synthesized by reaction of methyl 2-isothiocyanato benzoates $2\{1-6\}$ with 2 - $(\alpha, \beta, \omega$-aminoalkyl)imidazoles $3\{1-10\}$ in propan-2-ol solution with $\mathrm{KOH}$ as basic catalyst [Figure 2]. Formation of reaction products was controlled by TLC.

Screening of first time synthesized compounds for microbiological activity has been conducted in vitro by a generally accepted method of double serial dilution both in liquid and solid nutrient media. As biological target, the set of clinical and reference strains of 4 bacteria: Escherichia coli ATCC 25922 (F-50), Staphylococcus aureus ATCC 25923 (F-49), Bacillus anthracoides ATCC 1312, Pseudomonas aeruginosa ATCC 27853, and 1 fungi Candida albicans ATCC 885-653 have been used. Fluoroquinolone antibiotic Pipemidic acid was used as positive control for bacteria. Fluconazole was used as a positive fungal inhibitor standard for C. albicans.

\section{RESULTS AND DISCUSSION}

The structures of obtained compounds have been proved by analytical data [Table 1] and by hydrogen nuclear magnetic resonance ( ${ }^{1} \mathrm{H}$ NMR) spectroscopy data [Table 2]. Formation of the 2-thioxoquinazolin-4-one condensed system is confirmed by signals of NHCS protons at 13.20-12.78 ppm, while signals of $\mathrm{NH}$ protons of imidazole ring appear as a broad singlet at $14.83-13.54 \mathrm{ppm}$. Signals of $\mathrm{CH}=\mathrm{CH}$ protons of imidazole ring show at 7.10-7.22 ppm and 6.80-6.92 ppm. Position and multiplicity of signals of other protons are complied with the proposed structure of compounds.

Results of microbiological screening of 3-[2-(1H-imidazol2-yl)alkyl]-2-thioxo-2,3-dihydroquinazolin-4 $(1 \mathrm{H})$-one derivatives $4\{1-30\}$ are showed in Tables 3 and 4. The substances $4\{21\}-4\{28\}$ demonstrated the highest activity against $S$. aureus ATCC 25923 and C. albicans ATCC 885653. Considering the structure-activity relations can be noted the following regularities:

- Elongation of carbon chain between 2-thioxoquinazolin4-one and imidazole moieties up to 3 carbon atoms led to increasing of antimicrobial and antifungal action.

- The combination of 2-thioxoquinazolin-4-one and imidazole fragments in the molecules promotes the enhancement of activity against $S$. aureus.

\section{EXPERIMENTAL PART}

${ }^{1} \mathrm{H}$ NMR-spectra were recorded on Varian WXR-400 (200 MHz) spectrometer in Dimethyl sulfoxide (DMSO)- $d_{6}$

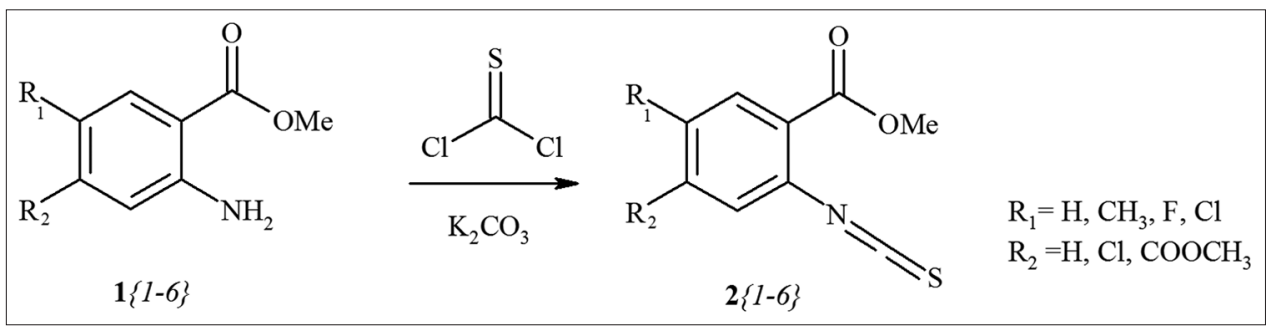

Figure 1: The scheme of methyl 2-isothiocyanato benzoates synthesis

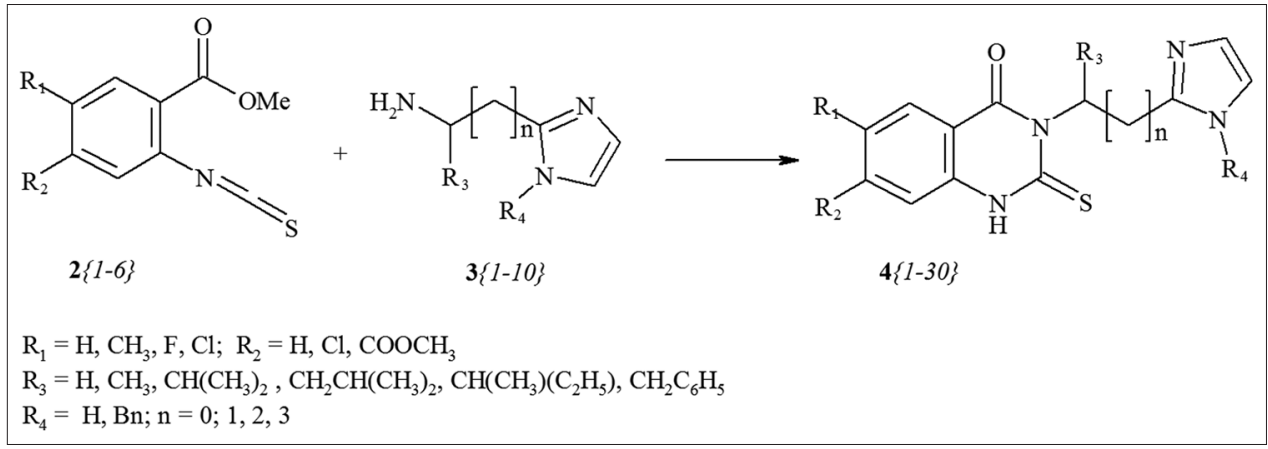

Figure 2: The scheme of 3-subsituted 2-thioxoquinazolin-4-ones synthesis 
Table 2: ${ }^{1} \mathrm{H}$ NMR spectra of 3-[2-(1H-imidazol-2-yl) alkyl]-2-thioxo-2,3-dihydroquinazolin-4 (1H)-ones 4\{1-30\}

\begin{tabular}{|c|c|}
\hline Compounds & ${ }^{1} \mathrm{H}$ NMR, $\delta$, p.p.m \\
\hline $4\{1\}$ & $\begin{array}{l}\text { 14.74-14.80 (br. s, } 1 \mathrm{H}, \mathrm{NH}), 12.78(\mathrm{~s}, 1 \mathrm{H}, \mathrm{NH}), 8.02(\mathrm{~d}, 1 \mathrm{H}, \mathrm{H}-5), 7.64(\mathrm{t}, 1 \mathrm{H}, \mathrm{H}-7), 7.45(\mathrm{~d}, 1 \mathrm{H}, \mathrm{H}-8) \text {, } \\
\text { 7.20-7.24 (m, 2H, H-6+CH), } 6.84(\mathrm{~s}, 1 \mathrm{H}, \mathrm{CH}), 4.28-4.32\left(\mathrm{t}, 2 \mathrm{H}, \mathrm{CH}_{2}\right)\end{array}$ \\
\hline $4\{2\}$ & $\begin{array}{l}\text { 14.80-14.83 (br. s, } 1 \mathrm{H}, \mathrm{NH}), 12.80(\mathrm{~s}, 1 \mathrm{H}, \mathrm{NH}), 8.00(\mathrm{~d}, 1 \mathrm{H}, \mathrm{H}-5), 7.66(\mathrm{t}, 1 \mathrm{H}, \mathrm{H}-7), 7.47(\mathrm{~d}, 1 \mathrm{H}, \mathrm{H}-8) \text {, } \\
7.22-7.25(\mathrm{~m}, 2 \mathrm{H}, \mathrm{H}-6+\mathrm{CH}), 6.82(\mathrm{~s}, 1 \mathrm{H}, \mathrm{CH}), 3.90-3.94\left(\mathrm{t}, 2 \mathrm{H}, \mathrm{NCH}_{2}\right), 2.06-2.10\left(\mathrm{t}, 2 \mathrm{H}, \mathrm{CH}_{2}\right)\end{array}$ \\
\hline $4\{3\}$ & $\begin{array}{l}\text { 13.80-13.83 (br. s, } 1 \mathrm{H}, \mathrm{NH}), 13.10(\mathrm{~s}, 1 \mathrm{H}, \mathrm{NH}), 7.88(\mathrm{~d}, 1 \mathrm{H}, \mathrm{H}-5), 7.68(\mathrm{t}, 1 \mathrm{H}, \mathrm{H}-7), 7.46(\mathrm{~d}, 1 \mathrm{H}, \mathrm{H}-8) \text {, } \\
7.28-7.33(\mathrm{~m}, 2 \mathrm{H}, \mathrm{H}-6+\mathrm{CH}), 6.82(\mathrm{~s}, 1 \mathrm{H}, \mathrm{CH}), 3.63\left(\mathrm{t}, 2 \mathrm{H}, \mathrm{NCH}_{2}\right), 2.18\left(\mathrm{t}, 2 \mathrm{H}, \mathrm{CH}_{2}\right), 1.78-1.82 \\
\left(\mathrm{~m}, 2 \mathrm{H}, \mathrm{CH}_{2}\right)\end{array}$ \\
\hline $4\{4\}$ & $\begin{array}{l}\text { 13.54-13.58 (br. s, } 1 \mathrm{H}, \mathrm{NH}), 12.82(\mathrm{~s}, 1 \mathrm{H}, \mathrm{NH}), 8.00(\mathrm{~d}, 1 \mathrm{H}, \mathrm{H}-5), 7.68(\mathrm{t}, 1 \mathrm{H}, \mathrm{H}-7), 7.47(\mathrm{~d}, 1 \mathrm{H}, \mathrm{H}-8) \text {, } \\
\text { 7.28-7.33 (m, } 2 \mathrm{H}, \mathrm{H}-6+\mathrm{CH}), 6.82(\mathrm{~s}, 1 \mathrm{H}, \mathrm{CH}), 3.60\left(\mathrm{t}, 2 \mathrm{H}, \mathrm{NCH}_{2}\right), 2.21\left(\mathrm{t}, 2 \mathrm{H}, \mathrm{CH}_{2}\right), 1.30-1.35 \\
\left(\mathrm{~m}, 4 \mathrm{H}, 2 \mathrm{CH}_{2}\right)\end{array}$ \\
\hline $4\{5\}$ & $\begin{array}{l}\text { 14.20-14.25 (br. s, 1H, NH), } 12.83(\mathrm{~s}, 1 \mathrm{H}, \mathrm{NH}), 8.02(\mathrm{~d}, 1 \mathrm{H}, \mathrm{H}-5), 7.66(\mathrm{t}, 1 \mathrm{H}, \mathrm{H}-7), 7.42(\mathrm{~d}, 1 \mathrm{H}, \mathrm{H}-8) \text {, } \\
7.24-7.27(\mathrm{~m}, 2 \mathrm{H}, \mathrm{H}-6+\mathrm{CH}), 6.92(\mathrm{~s}, 1 \mathrm{H}, \mathrm{CH}), 4.83-4.85(\mathrm{~m}, 1 \mathrm{H}, \mathrm{CH}), 1.52\left(\mathrm{~s}, 3 \mathrm{H}, \mathrm{CH}_{3}\right)\end{array}$ \\
\hline $4\{6\}$ & $\begin{array}{l}\text { 14.00-14.02 (br. s, } 1 \mathrm{H}, \mathrm{NH}), 12.83(\mathrm{~s}, 1 \mathrm{H}, \mathrm{NH}), 8.02(\mathrm{~d}, 1 \mathrm{H}, \mathrm{H}-5), 7.67(\mathrm{t}, 1 \mathrm{H}, \mathrm{H}-7), 7.44(\mathrm{~d}, 1 \mathrm{H}, \mathrm{H}-8) \\
7.20-7.25(\mathrm{~m}, 2 \mathrm{H}, \mathrm{H}-6+\mathrm{CH}), 6.92(\mathrm{~s}, 1 \mathrm{H}, \mathrm{CH}), 4.43-4.47(\mathrm{~m}, 1 \mathrm{H}, \mathrm{CH}), 2.50-2.54(\mathrm{~m}, 1 \mathrm{H}, \mathrm{CH}), 0.80 \\
\left(\mathrm{~s}, 6 \mathrm{H}, 2 \mathrm{CH}_{3}\right)\end{array}$ \\
\hline $4\{7\}$ & $\begin{array}{l}\text { 13.85-13.92 (br. s, } 1 \mathrm{H}, \mathrm{NH}), 12.93(\mathrm{~s}, 1 \mathrm{H}, \mathrm{NH}), 8.00(\mathrm{~d}, 1 \mathrm{H}, \mathrm{H}-5), 7.70(\mathrm{t}, 1 \mathrm{H}, \mathrm{H}-7), 7.44(\mathrm{~d}, 1 \mathrm{H}, \mathrm{H}-8) \text {, } \\
7.20-7.25(\mathrm{~m}, 2 \mathrm{H}, \mathrm{H}-6+\mathrm{CH}), 6.96(\mathrm{~s}, 1 \mathrm{H}, \mathrm{CH}), 4.74-4.77(\mathrm{~m}, 1 \mathrm{H}, \mathrm{CH}), 2.16-2.20\left(\mathrm{~m}, 2 \mathrm{H}, \mathrm{CH}_{2}\right) \\
\text { 1.79-1.82(m, } 1 \mathrm{H}, \mathrm{CH}), 0.90\left(\mathrm{~s}, 3 \mathrm{H}, \mathrm{CH}_{3}\right), 0.80\left(\mathrm{~s}, 3 \mathrm{H}, \mathrm{CH}_{3}\right)\end{array}$ \\
\hline $4\{8\}$ & $\begin{array}{l}\text { 13.88-13.94 (s, 1H, NH), } 13.03(\mathrm{~s}, 1 \mathrm{H}, \mathrm{NH}), 8.00(\mathrm{~d}, 1 \mathrm{H}, \mathrm{H}-5), 7.70(\mathrm{t}, 1 \mathrm{H}, \mathrm{H}-7), 7.42(\mathrm{~d}, 1 \mathrm{H}, \mathrm{H}-8) \text {, } \\
\text { 7.18-7.23 (m, 2H, H-6+CH), } 6.92(\mathrm{~s}, 1 \mathrm{H}, \mathrm{CH}), 4.48-4.51(\mathrm{~m}, 1 \mathrm{H}, \mathrm{NCH}), 1.43-1.46(\mathrm{~m}, \mathrm{H}, \mathrm{CH}) \\
1.38-1.40\left(\mathrm{~m}, 2 \mathrm{H}, \mathrm{CH}_{2}\right), 0.98\left(\mathrm{~s}, 3 \mathrm{H}, \mathrm{CH}_{3}\right), 0.80\left(\mathrm{~s}, 3 \mathrm{H}, \mathrm{CH}_{3}\right)\end{array}$ \\
\hline $4\{9\}$ & $\begin{array}{l}\text { 13.90-14.02 (br. s, H, NH), } 12.97(\mathrm{~s}, 1 \mathrm{H}, \mathrm{NH}), 8.02(\mathrm{~d}, 1 \mathrm{H}, \mathrm{H}-5), 7.74(\mathrm{t}, 1 \mathrm{H}, \mathrm{H}-7), 7.46(\mathrm{~d}, 1 \mathrm{H}, \mathrm{H}-8), \\
7.18-7.25(\mathrm{~m}, 7 \mathrm{H}, \mathrm{H}-6+\mathrm{CH}+\mathrm{Ar}-\mathrm{H}), 6.94(\mathrm{~s}, 1 \mathrm{H}, \mathrm{CH}), 5.02-5.10(\mathrm{~m}, 1 \mathrm{H}, \mathrm{CH}), 3.58-3.63\left(\mathrm{~m}, 2 \mathrm{H}, \mathrm{CH}_{2}\right)\end{array}$ \\
\hline $4\{10\}$ & $\begin{array}{l}13.02(\mathrm{~s}, 1 \mathrm{H}, \mathrm{NH}), 8.02(\mathrm{~d}, 1 \mathrm{H}, \mathrm{H}-5), 7.74(\mathrm{t}, 1 \mathrm{H}, \mathrm{H}-7), 7.46(\mathrm{~d}, 1 \mathrm{H}, \mathrm{H}-8), 7.18-7.25(\mathrm{~m}, 7 \mathrm{H}, \mathrm{H}-6+\mathrm{CH}+\mathrm{Ar}-\mathrm{H}) \text {, } \\
6.94(\mathrm{~s}, 1 \mathrm{H}, \mathrm{CH}), 5.18\left(\mathrm{~s}, 2 \mathrm{H}, \mathrm{NCH}_{2}\right), 3.75-3.70\left(\mathrm{t}, 2 \mathrm{H}, \mathrm{NCH}_{2}\right), 2.82-2.85\left(\mathrm{t}, 2 \mathrm{H}, \mathrm{CH}_{2}\right)\end{array}$ \\
\hline $4\{11\}$ & $\begin{array}{l}14.74-14.80(\mathrm{br} . \mathrm{s}, 1 \mathrm{H}, \mathrm{NH}), 12.78(\mathrm{~s}, 1 \mathrm{H}, \mathrm{NH}), 8.02(\mathrm{~d}, 1 \mathrm{H}, \mathrm{H}-5), 7.64(\mathrm{t}, 1 \mathrm{H}, \mathrm{H}-7), 7.45(\mathrm{~d}, 1 \mathrm{H}, \mathrm{H}-8) \\
7.10(\mathrm{~s}, 1 \mathrm{H}, \mathrm{CH}), 6.84(\mathrm{~s}, 1 \mathrm{H}, \mathrm{CH}), 4.28-4.32\left(\mathrm{t}, 2 \mathrm{H}, \mathrm{CH}_{2}\right), 2.35\left(\mathrm{~s}, 3 \mathrm{H}, \mathrm{CH}_{3}-\mathrm{Ar}\right)\end{array}$ \\
\hline $4\{12\}$ & $\begin{array}{l}14.56-14.60 \text { (br. s, } 1 \mathrm{H}, \mathrm{NH}), 12.78(\mathrm{~s}, 1 \mathrm{H}, \mathrm{NH}), 8.02(\mathrm{~d}, 1 \mathrm{H}, \mathrm{H}-5), 7.64(\mathrm{t}, 1 \mathrm{H}, \mathrm{H}-7), 7.45(\mathrm{~d}, 1 \mathrm{H}, \mathrm{H}-8) \\
7.10(\mathrm{~s}, 1 \mathrm{H}, \mathrm{CH}), 6.84(\mathrm{~s}, 1 \mathrm{H}, \mathrm{CH}), 3.90-3.94\left(\mathrm{t}, 2 \mathrm{H}, \mathrm{NCH}_{2}\right), 2.06-2.10\left(\mathrm{t}, 2 \mathrm{H}, \mathrm{CH}_{2}\right), 2.35\left(\mathrm{~s}, 3 \mathrm{H}, \mathrm{CH}_{3}-\mathrm{Ar}\right)\end{array}$ \\
\hline $4\{13\}$ & $\begin{array}{l}14.56-14.60(\text { br. s, } 1 \mathrm{H}, \mathrm{NH}), 12.78(\mathrm{~s}, 1 \mathrm{H}, \mathrm{NH}), 8.02(\mathrm{~d}, 1 \mathrm{H}, \mathrm{H}-5), 7.64(\mathrm{t}, 1 \mathrm{H}, \mathrm{H}-7), 7.47(\mathrm{~d}, 1 \mathrm{H}, \mathrm{H}-8) \\
7.10(\mathrm{~s}, 1 \mathrm{H}, \mathrm{CH}), 6.84(\mathrm{~s}, 1 \mathrm{H}, \mathrm{CH}), 3.90-3.94\left(\mathrm{t}, 2 \mathrm{H}, \mathrm{NCH}_{2}\right), 2.08-2.12\left(\mathrm{t}, 2 \mathrm{H}, \mathrm{CH}_{2}\right), 2.37\left(\mathrm{~s}, 3 \mathrm{H}, \mathrm{CH}_{3}-\mathrm{Ar}\right)\end{array}$ \\
\hline $4\{14\}$ & $\begin{array}{l}14.50-14.56(\text { br. s, } 1 \mathrm{H}, \mathrm{NH}), 12.80(\mathrm{~s}, 1 \mathrm{H}, \mathrm{NH}), 8.02(\mathrm{~d}, 1 \mathrm{H}, \mathrm{H}-5), 7.64(\mathrm{t}, 1 \mathrm{H}, \mathrm{H}-7), 7.45(\mathrm{~d}, 1 \mathrm{H}, \mathrm{H}-8) \\
7.10(\mathrm{~s}, 1 \mathrm{H}, \mathrm{CH}), 6.84(\mathrm{~s}, 1 \mathrm{H}, \mathrm{CH}), 3.63\left(\mathrm{t}, 2 \mathrm{H}, \mathrm{NCH}_{2}\right), 2.35\left(\mathrm{~s}, 3 \mathrm{H}, \mathrm{CH}_{3}-\mathrm{Ar}\right), 2.18\left(\mathrm{t}, 2 \mathrm{H}, \mathrm{CH}_{2}\right) \\
1.78-1.82\left(\mathrm{~m}, 2 \mathrm{H}, \mathrm{CH}_{2}\right)\end{array}$ \\
\hline $4\{15\}$ & $\begin{array}{l}\text { 13.78-13.82 (br. s, } 1 \mathrm{H}, \mathrm{NH}), 12.78(\mathrm{~s}, 1 \mathrm{H}, \mathrm{NH}), 8.02(\mathrm{~d}, 1 \mathrm{H}, \mathrm{H}-5), 7.64(\mathrm{t}, 1 \mathrm{H}, \mathrm{H}-7), 7.47(\mathrm{~d}, 1 \mathrm{H}, \mathrm{H}-8), \\
7.10(\mathrm{~s}, 1 \mathrm{H}, \mathrm{CH}), 6.86(\mathrm{~s}, 1 \mathrm{H}, \mathrm{CH}), 4.42-4.45(\mathrm{~m}, 1 \mathrm{H}, \mathrm{CH}), 2.53-2.56(\mathrm{~m}, 1 \mathrm{H}, \mathrm{CH}), 2.37\left(\mathrm{~s}, 3 \mathrm{H}, \mathrm{CH}_{3}-\mathrm{Ar}\right) \text {, } \\
0.82\left(\mathrm{~s}, 6 \mathrm{H}, 2 \mathrm{CH}_{3}\right)\end{array}$ \\
\hline $4\{16\}$ & $\begin{array}{l}13.80-13.84(\mathrm{br} . \mathrm{s}, 1 \mathrm{H}, \mathrm{NH}), 12.74(\mathrm{~s}, 1 \mathrm{H}, \mathrm{NH}), 8.02(\mathrm{~d}, 1 \mathrm{H}, \mathrm{H}-5), 7.62(\mathrm{t}, 1 \mathrm{H}, \mathrm{H}-7), 7.45(\mathrm{~d}, 1 \mathrm{H}, \mathrm{H}-8) \text {, } \\
7.10(\mathrm{~s}, 1 \mathrm{H}, \mathrm{CH}), 6.82(\mathrm{~s}, 1 \mathrm{H}, \mathrm{CH}), 4.80-4.85(\mathrm{~m}, 1 \mathrm{H}, \mathrm{CH}), 2.35\left(\mathrm{~s}, 3 \mathrm{H}, \mathrm{CH}_{3}-\mathrm{Ar}\right), 1.52\left(\mathrm{~s}, 6 \mathrm{H}, 2 \mathrm{CH}_{3}\right)\end{array}$ \\
\hline $4\{17\}$ & $\begin{array}{l}13.88-13.92(\text { br. s, } 1 \mathrm{H}, \mathrm{NH}), 12.80(\mathrm{~s}, 1 \mathrm{H}, \mathrm{NH}), 8.04(\mathrm{~d}, 1 \mathrm{H}, \mathrm{H}-5), 7.64(\mathrm{t}, 1 \mathrm{H}, \mathrm{H}-7), 7.47(\mathrm{~d}, 1 \mathrm{H}, \mathrm{H}-8) \\
7.10(\mathrm{~s}, 1 \mathrm{H}, \mathrm{CH}), 6.88(\mathrm{~s}, 1 \mathrm{H}, \mathrm{CH}), 5.42-5.45(\mathrm{~m}, 1 \mathrm{H}, \mathrm{CH}), 2.37\left(\mathrm{~s}, 3 \mathrm{H}, \mathrm{CH}_{3}-\mathrm{Ar}\right), 2.16-2.20\left(\mathrm{~m}, 2 \mathrm{H}, \mathrm{CH}_{2}\right), \\
1.76-1.80(\mathrm{~m}, 1 \mathrm{H}, \mathrm{CH}), 0.88\left(\mathrm{~s}, 6 \mathrm{H}, 2 \mathrm{CH}_{3}\right)\end{array}$ \\
\hline $4\{18\}$ & $\begin{array}{l}\text { 13.88-13.92 (br. s, } 1 \mathrm{H}, \mathrm{NH}), 12.76(\mathrm{~s}, 1 \mathrm{H}, \mathrm{NH}), 8.04(\mathrm{~d}, 1 \mathrm{H}, \mathrm{H}-5), 7.64(\mathrm{t}, 1 \mathrm{H}, \mathrm{H}-7), 7.48(\mathrm{~d}, 1 \mathrm{H}, \mathrm{H}-8) \text {, } \\
7.12(\mathrm{~s}, 1 \mathrm{H}, \mathrm{CH}), 6.88(\mathrm{~s}, 1 \mathrm{H}, \mathrm{CH}), 4.48-4.51(\mathrm{~m}, 1 \mathrm{H}, \mathrm{NCH}), 2.33\left(\mathrm{~s}, 3 \mathrm{H}, \mathrm{CH}_{3}-\mathrm{Ar}\right), 1.40-1.46(\mathrm{~m}, 3 \mathrm{H}, \\
\left.\mathrm{CH}+\mathrm{CH}_{2}\right), 0.98\left(\mathrm{~s}, 3 \mathrm{H}, \mathrm{CH}_{3}\right), 0.80\left(\mathrm{~s}, 3 \mathrm{H}, \mathrm{CH}_{3}\right)\end{array}$ \\
\hline $4\{19\}$ & $\begin{array}{l}\text { 13.90-14.02 (br. s, H, NH), } 12.85(\mathrm{~s}, 1 \mathrm{H}, \mathrm{NH}), 8.00(\mathrm{~d}, 1 \mathrm{H}, \mathrm{H}-5), 7.74(\mathrm{t}, 1 \mathrm{H}, \mathrm{H}-7), 7.48(\mathrm{~d}, 1 \mathrm{H}, \mathrm{H}-8) \text {, } \\
\text { 7.20-7.26 (m, } 6 \mathrm{H}, \mathrm{CH}+\mathrm{Ar}-\mathrm{H}), 6.94(\mathrm{~s}, 1 \mathrm{H}, \mathrm{CH}), 5.04-5.08(\mathrm{~m}, 1 \mathrm{H}, \mathrm{CH}), 3.60\left(\mathrm{~m}, 2 \mathrm{H}, \mathrm{CH}_{2}\right), 2.37 \\
\left(\mathrm{~s}, 3 \mathrm{H}, \mathrm{CH}_{3}-\mathrm{Ar}\right)\end{array}$ \\
\hline $4\{20\}$ & $\begin{array}{l}13.00(\mathrm{~s}, 1 \mathrm{H}, \mathrm{NH}), 8.02(\mathrm{~d}, 1 \mathrm{H}, \mathrm{H}-5), 7.74(\mathrm{t}, 1 \mathrm{H}, \mathrm{H}-7), 7.46(\mathrm{~d}, 1 \mathrm{H}, \mathrm{H}-8), 7.18-7.25(\mathrm{~m}, 6 \mathrm{H}, \mathrm{CH}+\mathrm{Ar}-\mathrm{H}), \\
6.94(\mathrm{~s}, 1 \mathrm{H}, \mathrm{CH}), 5.18\left(\mathrm{~s}, 2 \mathrm{H}, \mathrm{NCH}_{2}\right), 3.72-3.78\left(\mathrm{t}, 2 \mathrm{H}, \mathrm{NCH}_{2}\right), 2.82-2.85\left(\mathrm{t}, 2 \mathrm{H}, \mathrm{CH}_{2}\right), 2.34(\mathrm{~s}, 3 \mathrm{H}, \mathrm{CH}-\mathrm{Ar})\end{array}$ \\
\hline $4\{21\}$ & $\begin{array}{l}14.00-14.04 \text { (br. s, H, NH), } 12.93(\mathrm{~s}, 1 \mathrm{H}, \mathrm{NH}), 7.60-7.80(\mathrm{~m}, 2 \mathrm{H}, \mathrm{H}-5,7), 7.12(\mathrm{~s}, 1 \mathrm{H}, \mathrm{CH}), 7.33 \\
(\mathrm{qr}, 1 \mathrm{H}, \mathrm{H}-8), 6.88(\mathrm{~s}, 1 \mathrm{H}, \mathrm{CH}), 3.88\left(\mathrm{t}, 2 \mathrm{H}, \mathrm{CH}_{2}\right), 2.10\left(\mathrm{t}, 2 \mathrm{H}, \mathrm{CH}_{2}\right)\end{array}$ \\
\hline
\end{tabular}




\begin{tabular}{|c|c|}
\hline Compounds & ${ }^{1} \mathrm{H}$ NMR, $\delta$, p.p.m \\
\hline $4\{22\}$ & $\begin{array}{l}13.78-13.81 \text { (br. s, } 1 \mathrm{H}, \mathrm{NH}), 12.95(\mathrm{~s}, 1 \mathrm{H}, \mathrm{NH}), 7.62-7.78(\mathrm{~m}, 2 \mathrm{H}, \mathrm{H}-5,7), 7.14(\mathrm{~s}, 1 \mathrm{H}, \mathrm{CH}), 7.33 \\
(\mathrm{qr}, 1 \mathrm{H}, \mathrm{H}-8), 6.88(\mathrm{~s}, 1 \mathrm{H}, \mathrm{CH}), 3.65\left(\mathrm{t}, 2 \mathrm{H}, \mathrm{CH}_{2}\right), 2.16\left(\mathrm{t}, 2 \mathrm{H}, \mathrm{CH}_{2}\right), 1.80-1.84\left(\mathrm{~m}, 2 \mathrm{H}, \mathrm{CH}_{2}\right)\end{array}$ \\
\hline $4\{23\}$ & $\begin{array}{l}13.52-13.56 \text { (br. s, } 1 \mathrm{H}, \mathrm{NH}), 12.90(\mathrm{~s}, 1 \mathrm{H}, \mathrm{NH}), 7.60-7.76(\mathrm{~m}, 2 \mathrm{H}, \mathrm{H}-5,7), 7.12(\mathrm{~s}, 1 \mathrm{H}, \mathrm{CH}), 7.33 \\
(\mathrm{qr}, 1 \mathrm{H}, \mathrm{H}-8), 6.88(\mathrm{~s}, 1 \mathrm{H}, \mathrm{CH}), 3.60\left(\mathrm{t}, 2 \mathrm{H}, \mathrm{CH}_{2}\right), 2.22-2.26\left(\mathrm{t}, 2 \mathrm{H}, \mathrm{CH}_{2}\right), 1.30-1.34\left(\mathrm{~m}, 4 \mathrm{H}, 2 \mathrm{CH}_{2}\right)\end{array}$ \\
\hline $4\{24\}$ & $\begin{array}{l}13.00(\mathrm{~s}, 1 \mathrm{H}, \mathrm{NH}), 8.02(\mathrm{~d}, 1 \mathrm{H}, \mathrm{H}-5), 7.74(\mathrm{t}, 1 \mathrm{H}, \mathrm{H}-7), 7.46(\mathrm{~d}, 1 \mathrm{H}, \mathrm{H}-8), 7.18-7.25(\mathrm{~m}, 6 \mathrm{H}, \mathrm{CH}+\mathrm{Ar}-\mathrm{H}) \text {, } \\
6.94(\mathrm{~s}, 1 \mathrm{H}, \mathrm{CH}), 5.18\left(\mathrm{~s}, 2 \mathrm{H}, \mathrm{NCH}_{2}\right), 3.72-3.78\left(\mathrm{t}, 2 \mathrm{H}, \mathrm{NCH}_{2}\right), 2.82-2.85\left(\mathrm{t}, 2 \mathrm{H}, \mathrm{CH}_{2}\right)\end{array}$ \\
\hline $4\{25\}$ & $\begin{array}{l}14.82(\mathrm{~s}, 1 \mathrm{H}, \mathrm{NH}), 12.95(\mathrm{~s}, 1 \mathrm{H}, \mathrm{NH}), 7.87(\mathrm{~s}, 1 \mathrm{H}, \mathrm{H}-5), 7.75(\mathrm{~d}, 1 \mathrm{H}, \mathrm{H}-7), 7.20(\mathrm{~s}, \mathrm{H}, \mathrm{CH}), 7.38 \\
(\mathrm{~d}, 1 \mathrm{H}, \mathrm{H}-8), 6.86(\mathrm{~s}, \mathrm{H}, \mathrm{CH}), 3.94\left(\mathrm{t}, 2 \mathrm{H}, \mathrm{CH}_{2}\right), 2.12\left(\mathrm{t}, 2 \mathrm{H}, \mathrm{CH}_{2}\right)\end{array}$ \\
\hline $4\{26\}$ & $\begin{array}{l}13.78-13.82(\mathrm{br} . \mathrm{s}, 1 \mathrm{H}, \mathrm{NH}), 12.97(\mathrm{~s}, 1 \mathrm{H}, \mathrm{NH}), 7.89(\mathrm{~s}, 1 \mathrm{H}, \mathrm{H}-5), 7.75(\mathrm{~d}, 1 \mathrm{H}, \mathrm{H}-7), 7.22(\mathrm{~s}, \mathrm{H}, \mathrm{CH}) \\
7.38(\mathrm{~d}, 1 \mathrm{H}, \mathrm{H}-8), 6.86(\mathrm{~s}, \mathrm{H}, \mathrm{CH}), 3.64\left(\mathrm{t}, 2 \mathrm{H}, \mathrm{CH}_{2}\right), 2.18\left(\mathrm{t}, 2 \mathrm{H}, \mathrm{CH}_{2}\right), 1.78-1.80\left(\mathrm{~m}, 2 \mathrm{H}, \mathrm{CH}_{2}\right)\end{array}$ \\
\hline $4\{27\}$ & $\begin{array}{l}13.56-13.60(\text { br. s, } 1 \mathrm{H}, \mathrm{NH}), 12.90(\mathrm{~s}, 1 \mathrm{H}, \mathrm{NH}), 7.95(\mathrm{~d}, 1 \mathrm{H}, \mathrm{H}-5), 7.24-7.32(\mathrm{~m}, 2 \mathrm{H}, \mathrm{H}-6,8), 7.18 \\
(\mathrm{~s}, \mathrm{H}, \mathrm{CH}), 6.90(\mathrm{~s}, \mathrm{H}, \mathrm{CH}), 3.04\left(\mathrm{t}, 2 \mathrm{H}, \mathrm{CH}_{2}\right), 2.22\left(\mathrm{t}, 2 \mathrm{H}, \mathrm{CH}_{2}\right), 1.32-1.36\left(\mathrm{~m}, 4 \mathrm{H}, 2 \mathrm{CH}_{2}\right)\end{array}$ \\
\hline $4\{28\}$ & $\begin{array}{l}13.00(\mathrm{~s}, 1 \mathrm{H}, \mathrm{NH}), 7.98(\mathrm{~d}, 1 \mathrm{H}, \mathrm{H}-5), 7.26-7.35(\mathrm{~m}, 2 \mathrm{H}, \mathrm{H}-6,8), 7.18-7.22(\mathrm{~m}, 6 \mathrm{H}, \mathrm{CH}+\mathrm{Ar}-\mathrm{H}), 6.94 \\
(\mathrm{~s}, 1 \mathrm{H}, \mathrm{CH}), 5.16\left(\mathrm{~s}, 2 \mathrm{H}, \mathrm{NCH}_{2}\right), 3.72-3.75\left(\mathrm{t}, 2 \mathrm{H}, \mathrm{NCH}_{2}\right), 2.80-2.83\left(\mathrm{t}, 2 \mathrm{H}, \mathrm{CH}_{2}\right)\end{array}$ \\
\hline $4\{29\}$ & $\begin{array}{l}14.81(\mathrm{~s}, 1 \mathrm{H}, \mathrm{NH}), 13.20(\mathrm{~s}, 1 \mathrm{H}, \mathrm{NH}), 8.00(\mathrm{~d}, 1 \mathrm{H}, \mathrm{H}-5), 7.78(\mathrm{~s}, 1 \mathrm{H}, \mathrm{H}-8), 7.66(\mathrm{~d}, 1 \mathrm{H}, \mathrm{H}-6), 7.18 \\
(\mathrm{~s}, \mathrm{H}, \mathrm{CH}), 7.38(\mathrm{~d}, 1 \mathrm{H}, \mathrm{H}-8), 6.84(\mathrm{~s}, \mathrm{H}, \mathrm{CH}), 3.96\left(\mathrm{t}, 2 \mathrm{H}, \mathrm{CH}_{2}\right), 3.90\left(\mathrm{~s}, 3 \mathrm{H}, \mathrm{OCH}_{3}\right), 2.12\left(\mathrm{t}, 2 \mathrm{H}, \mathrm{CH}_{2}\right)\end{array}$ \\
\hline $4\{30\}$ & $\begin{array}{l}13.80-13.85(\text { br. s, } 1 \mathrm{H}, \mathrm{NH}), 13.20(\mathrm{~s}, 1 \mathrm{H}, \mathrm{NH}), 8.00(\mathrm{~d}, 1 \mathrm{H}, \mathrm{H}-5), 7.78(\mathrm{~s}, 1 \mathrm{H}, \mathrm{H}-8), 7.66(\mathrm{~d}, 1 \mathrm{H}, \mathrm{H}-6) \\
7.18(\mathrm{~s}, \mathrm{H}, \mathrm{CH}), 7.38(\mathrm{~d}, 1 \mathrm{H}, \mathrm{H}-8), 6.84(\mathrm{~s}, \mathrm{H}, \mathrm{CH}), 3.90\left(\mathrm{~s}, 3 \mathrm{H}, \mathrm{OCH}_{3}\right), 3.64\left(\mathrm{t}, 2 \mathrm{H}, \mathrm{CH}_{2}\right), 2.18\left(\mathrm{t}, 2 \mathrm{H}, \mathrm{CH}_{2}\right) \text {, } \\
1.74-1.78\left(\mathrm{~m}, 2 \mathrm{H}, \mathrm{CH}_{2}\right)\end{array}$ \\
\hline
\end{tabular}

H NMR: Hydrogen nuclear magnetic resonance

using TMS as an internal standard (chemical shifts are reported in ppm). Elemental analysis was performed on Euro EA-3000 apparatus. Melting points were measured with a Buchi B-520 melting point apparatus and were not corrected. Thin-layer chromatography was performed on Silufol UV 254 aluminum plates precoated with silica gel. Ethyl acetate was used as eluent.

Chemicals and solvents were of analytical grade. Methyl 2-aminobenzoate 99\% purity (CAS Number 134-20-3), methyl 2-amino-5-chlorobenzoate 95\% purity (CAS Number 5202-89-1), dimethyl 2-aminoterephtalate 97\% purity (CAS Number 5372-81-6), thiophosgene $97 \%$ purity (CAS Number 463-71-8), and solvents were purchased from Sigma-Aldrich, methyl 2-amino-5-methylbenzoate $98 \%$ purity (CAS Number 18595-16-9), methyl 2-amino-5-fluorobenzoate 98\% purity (CAS Number 319-24-4), and methyl 2-amino-4chlorobenzoate $98 \%$ purity (CAS Number 5900-58-3) were purchased from Enamine, Kyiv, Ukraine.

The Synthesis of methyl 2-isothiocyanato benzoates $2\{1-6\}$. To stirred mixture of $100 \mathrm{ml}$ of chloroform and $50 \mathrm{ml}$ of water $0.1 \mathrm{~mol}$ of corresponding substituted methyl 2 -aminobenzoates $1\{1-6\}$ was added. Then, the solution of $12.65 \mathrm{~g}(0.11 \mathrm{~mol})$ of thiophosgene in $50 \mathrm{ml}$ of chloroform was added dropwise with effective stirring in such a way that temperature does not exceed $25^{\circ} \mathrm{C}$. The reaction mixture was stirred at room temperature for 2-3 h and solution of $34.5 \mathrm{~g}$ $(0.25 \mathrm{~mol})$ of $\mathrm{K}_{2} \mathrm{CO}_{3}$ in $50 \mathrm{ml}$ of water was added. Lower organic layer was separated, washed 3 times of $100 \mathrm{ml}$ of water and dried with $\mathrm{MgSO}_{4}$. Chloroform was evaporated under lowered pressure. Obtained methyl 2-isothiocyanato benzoates $2\{1-6\}$ was used for next stage of synthesis without additional purification.

- Methyl 2-isothiocyanato benzoate 2\{1\}: Yield $86 \%$, m.p. $97^{\circ} \mathrm{C}$, m.p. $98^{\circ} \mathrm{C}[24]$.

- Methyl 2-isothiocyanato-5-methylbenzoate 2\{2\}: Yield $93 \%$, m.p. $96^{\circ} \mathrm{C}$.

- Methyl 2-isothiocyanato-5-fluorobenzoate 2\{3\}: Yield $83 \%$, m.p. $102^{\circ} \mathrm{C}$.

- Methyl 2-isothiocyanato-5-chlorobenzoate 2\{4\}: Yield $79 \%$, m.p. $81^{\circ} \mathrm{C}$.

- Methyl 2-isothiocyanato-4-chlorobenzoate 2\{5\}: Yield $86 \%$, m.p. $91^{\circ} \mathrm{C}$.

- Dimethyl 2-isothiocyanato terephthalate 2\{6\}: Yield $88 \%$, m.p. $109^{\circ} \mathrm{C}$.

The synthesis of 3-[2-(1H-imidazol-2-yl)alkyl]-2-thioxo2,3-dihydroquinazolin-4(1H)-ones $4\{1-30\}$. Corresponding methyl 2-isothiocyanatobenzoate $2\{1-6\}(0.05 \mathrm{~mol})$ was dissolved in $40 \mathrm{ml}$ of propanol-2. Then, corresponding $2-(\alpha, \beta, \omega$-aminoalkyl)imidazole $3\{1-10\} \quad(0.055 \mathrm{~mol})$ was added slowly and $6 \mathrm{ml}$ of $50 \%$ water solution of $\mathrm{KOH}$ was added dropwise. The reaction mixture was refluxed for 30 minutes, cooled to room temperature, poured into $100 \mathrm{ml}$ of water and neutralized by acetic acid to $\mathrm{pH}=7$. Formed precipitate was filtered and crystallized from a mixture of DMF and propanol-2 (1:1). Yields of obtained compounds $4\{1-30\}$ are given in Table 1 .

\section{Experimental Biological Part}

Samples of each compound were dissolved in DMSO that gave $1 \mathrm{mg} / \mathrm{ml}$ solution just before screening. Hottinger broth 


\begin{tabular}{|c|c|c|c|c|c|c|}
\hline \multirow[t]{2}{*}{ Compounds } & \multicolumn{2}{|c|}{ S. aureus ATCC 25923} & \multicolumn{2}{|c|}{ Escherichia coli ATCC 25922} & \multicolumn{2}{|c|}{ P. aeruginosa ATCC 27853} \\
\hline & $\mathrm{MIC} \mu \mathrm{g} / \mathrm{ml}$ & $\mathrm{MBC} \mu \mathrm{g} / \mathrm{ml}$ & $\mathrm{MIC} \mu \mathrm{g} / \mathrm{ml}$ & $\mathrm{MBC} \mu \mathrm{g} / \mathrm{ml}$ & $\mathrm{MIC} \mu \mathrm{g} / \mathrm{ml}$ & $\mathrm{MBC} \mu \mathrm{g} / \mathrm{ml}$ \\
\hline Pipemidic acid & 6.25 & & 25.00 & & 12.50 & \\
\hline $4\{1\}$ & 50.0 & 100.0 & 50.0 & 200.0 & 50.0 & 100.0 \\
\hline $4\{2\}$ & 25.0 & 100.0 & 100.0 & 200.0 & 50.0 & 200.0 \\
\hline $4\{3\}$ & 50.0 & 100.0 & 25.0 & 50.0 & 50.0 & 100.0 \\
\hline $4\{4\}$ & 50.0 & 100.0 & 50.0 & 25.0 & 50.0 & 25.0 \\
\hline $4\{5\}$ & 50.0 & 100.0 & 50.0 & 100.0 & 50.0 & 100.0 \\
\hline $4\{6\}$ & 50.0 & 100.0 & 50.0 & 200.0 & 50.0 & 100.0 \\
\hline $4\{7\}$ & 25.0 & 100.0 & 100.0 & 200.0 & 50.0 & 200.0 \\
\hline $4\{8\}$ & 50.0 & 100.0 & 50.0 & 200.0 & 100.0 & 200.0 \\
\hline $4\{9\}$ & 50.0 & 100.0 & 50.0 & 100.0 & 50.0 & 100.0 \\
\hline $4\{10\}$ & 50.0 & 100.0 & 50.0 & 100.0 & 50.0 & 100.0 \\
\hline $4\{11\}$ & 50.0 & 100.0 & 50.0 & 200.0 & 50.0 & 100.0 \\
\hline $4\{12\}$ & 50.0 & 100.0 & 100.0 & 200.0 & 50.0 & 200.0 \\
\hline $4\{13\}$ & 50.0 & 100.0 & 50.0 & 200.0 & 50.0 & 100.0 \\
\hline $4\{14\}$ & 50.0 & 100.0 & 25.0 & 50.0 & 50.0 & 100.0 \\
\hline $4\{15\}$ & 50.0 & 100.0 & 50.0 & 200.0 & 50.0 & 100.0 \\
\hline $4\{16\}$ & 25.0 & 50.0 & 100.0 & 200.0 & 50.0 & 200.0 \\
\hline $4\{17\}$ & 50.0 & 100.0 & 50.0 & 200.0 & 100.0 & 200.0 \\
\hline $4\{18\}$ & 50.0 & 100.0 & 50.0 & 100.0 & 50.0 & 100.0 \\
\hline $4\{19\}$ & 50.0 & 100.0 & 50.0 & 100.0 & 50.0 & 200.0 \\
\hline $4\{20\}$ & 50.0 & 100.0 & 50.0 & 200.0 & 50.0 & 100.0 \\
\hline $4\{21\}$ & 25.0 & 50.0 & 50.0 & 100.0 & 50.0 & 100.0 \\
\hline $4\{22\}$ & 25.0 & 50.0 & 25.0 & 100.0 & 50.0 & 100.0 \\
\hline $4\{23\}$ & 25.0 & 50.0 & 50.0 & 100.0 & 50.0 & 100.0 \\
\hline $4\{24\}$ & 25.0 & 50.0 & 25.0 & 50.0 & 50.0 & 100.0 \\
\hline $4\{25\}$ & 25.0 & 50.0 & 50.0 & 100.0 & 50.0 & 100.0 \\
\hline $4\{26\}$ & 25.0 & 50.0 & 50.0 & 100.0 & 50.0 & 100.0 \\
\hline $4\{27\}$ & 25.0 & 50.0 & 25.0 & 50.0 & 50.0 & 25.0 \\
\hline $4\{28\}$ & 50.0 & 100.0 & 50.0 & 100.0 & 50.0 & 100.0 \\
\hline $4\{29\}$ & 50.0 & 200.0 & 50.0 & 100.0 & 50.0 & 100.0 \\
\hline $4\{30\}$ & 50.0 & 100.0 & 50.0 & 100.0 & 50.0 & 100.0 \\
\hline
\end{tabular}

MIC: Minimum inhibitor concentration, S. aureus: Staphylococcus aureus, $P$. aeruginosa: Pseudomonas aeruginosa

( $\mathrm{pH} 7.2-7.4)$, which was used as a nutrition medium for bacteria, was poured at $2 \mathrm{ml}$ into 10 sterile test tubes. Then, $2 \mathrm{ml}$ of the solution of the tested compound was added into first test tube. The mixture was thoroughly stirred, and $2 \mathrm{ml}$ of obtained solution was added into the next test tube. The procedure was repeated until penultimate tube, from which $2 \mathrm{ml}$ of the resulted mixture was poured out. The last tube with $2 \mathrm{ml}$ of nutrition medium was used as the control. Thus, consecutive dilutions of the solution of tested compound at concentrations ranging from 400 to $2 \mu \mathrm{g} / \mathrm{ml}$ were obtained. Then, tested microbial cultures of $2 \times 10^{5} \mathrm{CFU} / \mathrm{ml}$ were added, and test tubes were incubated at $37^{\circ} \mathrm{C}$ for $18-24 \mathrm{~h}$. Fungi strains were cultured in Sabouraud medium with microbial density $2 \times 10^{5} \mathrm{CFU} / \mathrm{ml}$; the test tubes were incubated at $30^{\circ} \mathrm{C}$ for $48 \mathrm{~h}$. Minimum inhibitory concentration (MIC) was determined by the absence of apparent growth of microorganisms in a liquid nutrient medium. The medium remained transparent in those test tubes, where the concentration of the tested compound was sufficient to completely suppress the growth of the test microorganism. The turbidity of the samples indicates that the concentration of the substance in them is less than the MIC for this test culture. Minimum bactericidal concentration 


\begin{tabular}{|c|c|c|c|c|c|c|}
\hline \multirow[t]{2}{*}{ Compounds } & \multicolumn{2}{|c|}{ P. vulgaris ATCC 4636} & \multicolumn{2}{|c|}{ B. anthracoides ATCC 1312} & \multicolumn{2}{|c|}{ C. albicans ATCC $885-653$} \\
\hline & MIC $\mu \mathrm{g} / \mathrm{ml}$ & $\mathrm{MBC} \mu \mathrm{g} / \mathrm{ml}$ & MIC $\mu \mathrm{g} / \mathrm{ml}$ & MBC $\mu \mathrm{g} / \mathrm{ml}$ & MIC $\mu \mathrm{g} / \mathrm{ml}$ & MBC $\mu \mathrm{g} / \mathrm{ml}$ \\
\hline Pipemidic acid & 12.5 & & 12.5 & & & \\
\hline Fluconazole & & & & & 15.6 & 62.5 \\
\hline $4\{1\}$ & 50.0 & 200.0 & 50.0 & 100.0 & 100.0 & 200.0 \\
\hline $4\{2\}$ & 100.0 & 200.0 & 50.0 & 200.0 & 50.0 & 100.0 \\
\hline $4\{3\}$ & 25.0 & 50.0 & 25.0 & 50.0 & 25.0 & 50.0 \\
\hline $4\{4\}$ & 50.0 & 50.0 & 50.0 & 25.0 & 50.0 & 25.0 \\
\hline $4\{5\}$ & 50.0 & 200.0 & 50.0 & 100.0 & 50.0 & 100.0 \\
\hline $4\{6\}$ & 50.0 & 200.0 & 50.0 & 200.0 & 100.0 & 200.0 \\
\hline $4\{7\}$ & 50.0 & 100.0 & 50.0 & 200.0 & 50.0 & 100.0 \\
\hline $4\{8\}$ & 50.0 & 200.0 & 50.0 & 100.0 & 50.0 & 200.0 \\
\hline $4\{9\}$ & 50.0 & 200.0 & 50.0 & 100.0 & 50.0 & 100.0 \\
\hline $4\{10\}$ & 50.0 & 200.0 & 50.0 & 200.0 & 50.0 & 100.0 \\
\hline $4\{11\}$ & 50.0 & 200.0 & 50.0 & 100.0 & 100.0 & 200.0 \\
\hline $4\{12\}$ & 50.0 & 100.0 & 50.0 & 200.0 & 100.0 & 200.0 \\
\hline $4\{13\}$ & 50.0 & 200.0 & 50.0 & 100.0 & 100.0 & 200.0 \\
\hline $4\{14\}$ & 50.0 & 100.0 & 25.0 & 50.0 & 25.0 & 50.0 \\
\hline $4\{15\}$ & 50.0 & 200.0 & 50.0 & 100.0 & 100.0 & 200.0 \\
\hline $4\{16\}$ & 50.0 & 100.0 & 50.0 & 200.0 & 50.0 & 100.0 \\
\hline $4\{17\}$ & 50.0 & 200.0 & 50.0 & 100.0 & 50.0 & 50.0 \\
\hline $4\{18\}$ & 50.0 & 200.0 & 50.0 & 100.0 & 50.0 & 100.0 \\
\hline $4\{19\}$ & 50.0 & 200.0 & 50.0 & 100.0 & 50.0 & 100.0 \\
\hline $4\{20\}$ & 50.0 & 200.0 & 50.0 & 100.0 & 100.0 & 200.0 \\
\hline $4\{21\}$ & 25.0 & 100.0 & 50.0 & 100.0 & 25.0 & 50.0 \\
\hline $4\{22\}$ & 50.0 & 100.0 & 50.0 & 100.0 & 25.0 & 50.0 \\
\hline $4\{23\}$ & 25.0 & 50.0 & 50.0 & 100.0 & 25.0 & 50.0 \\
\hline $4\{24\}$ & 50.0 & 100.0 & 50.0 & 100.0 & 25.0 & 50.0 \\
\hline $4\{25\}$ & 25.0 & 50.0 & 50.0 & 100.0 & 50.0 & 100.0 \\
\hline $4\{26\}$ & 50.0 & 100.0 & 50.0 & 100.0 & 50.0 & 100.0 \\
\hline $4\{27\}$ & 25.0 & 50.0 & 25.0 & 50.0 & 25.0 & 50.0 \\
\hline $4\{28\}$ & 50.0 & 100.0 & 50.0 & 100.0 & 100.0 & 200.0 \\
\hline $4\{29\}$ & 50.0 & 200.0 & 50.0 & 100.0 & 50.0 & 100.0 \\
\hline $4\{30\}$ & 50.0 & 200.0 & 50.0 & 100.0 & 50.0 & 100.0 \\
\hline
\end{tabular}

MIC: Minimum inhibitor concentration, MBC: Minimum bactericidal concentration, $P$. vulgaris: Proteus vulgaris, $B$. anthracoidesm: Bacillus anthracoidesm, C. albicans: Candida albicans

(MBC) was determined by seeding microorganisms from samples on a solid nutrient medium.

\section{CONCLUSIONS}

The series of novel 6,7-substituted 3-[2-(1H-imidazol-2-yl) alkyl]-2-thioxo-2,3-dihydroquinazolin-4(1H)-ones, which contain 2-thioxoquinazolin-4-one and imidazole moieties connected by carbon chain have been synthesized.

Antibacterial and antifungal activity of novel 6,7-substituted 3-[2-(1H-imidazol-2-yl)alkyl]-2-thioxo-2,3dihydroquinazolin-4 $(1 H)$-one derivatives has been studied by the method of double serial dilution with the standard set of microbial strains as a biological target. 
Elongation of carbon chain between 2-thioxoquinazolin-4one and imidazole moieties up to three carbon atoms led to increasing of antimicrobial and antifungal action.

\section{REFERENCES}

1. Shalini K, Sharma PK, Kumar N. Imidazole and its biological activities: A review. Chem Sin 2010;1:36-47.

2. Bhatnagar A, Sharma PK, Kumar N. A review on "imidazoles": Their chemistry and pharma-cological potentials. Int J PharmTech Res 2011;3:268-82.

3. Vijayakumar B, Prasanthi P, Teja MK, Reddy MK, Nishanthi M, Nagendramma M, et al. Quinazoline derivatives and pharmacological activities. A review. Int J Med Chem Anal 2013;3:10-21.

4. Kubicová L, Ustr M, Král'ová K, Chobot V, Jahodar L, Machacek M, et al. Synthesis and biological evaluation of quinazoline-4-thiones. Molecules 2003;8:756-69.

5. Jantová S, Stankovský Š, Špirková K. In vitro antibacterial activity of ten series of substituted quinazolines. Biologia 2004;59:741-52.

6. Taltavull J, Serrat J, Gràcia J, Gavalda A, Andres M, Cordoba M, et al. Synthesis and biological activity of pyrido[3',2':4,5] thieno[3,2-d]pyrimidines as phosphodiesterase type 4 inhibitors. J Med Chem 2010;53:6912-22.

7. Kumaraswamy D, Mallareddy V. Synthesis and biological activities of some s-mannich bases of 1,4,5,6,7,8-hexahydro quinazolines. Res J Pharm Biol Chem Sci 2011;2:333-40.

8. Chaudhary RP. Synthesis and antimicrobial studies of some thiazolo [2,3-b]benzo[f]quinazoline and thiazino[2,3-b]benzo[f]quinazoline derivatives. Der Pharma Chem 2011;3:288-92.

9. Wang D, Gao F. Quinazoline derivatives: Synthesis and bioactivities. Chem Cent J 2013;7:95.

10. Bodke Y, Sangapure SS. Synthesis and biological activity of some benzofuro[3,2-d]pyrimidines. J Indian Chem Soc 2003;80:187-9.

11. Lagunin AA, Stepanchikova AV, Filimonov DA, Poroikov VV. PASS: Prediction of activity spectra for biologically active substances. Bioinformatics 2000;16:747-8.

12. Ivachtchenko A, Kovalenko $\mathrm{S}$, Tkachenko OV, Parkhomenko O. Synthesis of substituted thienopyrimidine4-ones. J Comb Chem 2004;6:573-83.

13. Tkachenko EV, Vlasov SV, Zhuravel IA, Kovalenko SN, Chernych VP. Synthesis and antimicrobial action 2-alkiltio-3-N- substituted tieno[3,2-d]pirimidin4(3H)-onov. Nauchnyie vedomosti BelGU. Med Farmatsiya 2013;25:131-8.

14. Ilchenko OV, Zaremba OV, Sheryakov AA. Synthesis 2-[(3-substituted-4-okso-3,4-dihidro[1]benzofuro[3,2-d] pirimidin-2-il)sulfanil]-N-alkil(aril) $\neg$ atseta $\neg$ midiv and their antimicrobial action. Zh Med Khim 2007;1:91-5.

15. Ivachtchenko AV, Kovalenko SM, Drushlyak OG. Synthesis of substituted 4-oxo-2-thioxo-1,2,3,4tetrahydroquinazolinesand4-oxo-3,4-dihydroquinazoline2-thioles. J Comb Chem 2003;5:775-88.

16. Borisov OV, Zavada OO, Zhuravel IO, Kovalenko SM. Strukturna modifIkatsIya amInokislot: Sintez 2-( $\alpha, \beta, \omega-$ aminoalkil)imidazoliv. J Org Pharm Chem 2013;1:66-9.

17. Kovalenko SM, Zhuravel IO, Borisov OV, Zavada OO. Sposib Oderzhannya Zamischenih 2-( $\alpha, \beta, \omega$-aminoalkil) Imidazoliv. Publ. 25.07.2014. Bul N 14. Patent No. 106083 UA, MPK C07D 233/54 (2006.01); MPK C07D 233/64 (2006.01).

Source of Support: The research work performed in the manuscript is done on the funding of National University of Pharmacy (Ukraine). Conflict of Interest: None declared. 